\title{
A Comparison between Lattice-Valued Propositional Logic LP(X) and Gradational Lattice-Valued Propositional Logic $\mathbf{L}_{\mathbf{v p l}}$
}

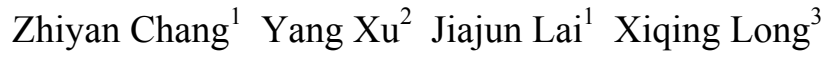 \\ ${ }^{1}$ Intelligent Control Development Center, Southwest Jiaotong University, Chengdu 610031, P. R. China \\ ${ }^{2}$ Department of Mathematics, Southwest Jiaotong University, Chengdu 610031, P. R. China \\ ${ }^{3}$ Department of Mathematics, Yibin University, Yibin 644007, P. R. China
}

\begin{abstract}
By comparing the definitions of $\mathrm{L}_{\mathrm{vpl}}$ with the corresponding definitions of $\mathrm{LP}(\mathrm{X})$, the meaning of the parameters of $\mathrm{L}_{\mathrm{vpl}}$ and the role of inference rule set $\Re$ playing in uncertainty reasoning based on $\mathrm{L}_{\mathrm{vpl}}$ are demonstrated. And to some extent it is shown that the choose of $R$ determines the semantic and syntactic function of uncertainty reasoning based on $\mathrm{L}_{\mathrm{vpl}}$.
\end{abstract}

Keywords: Lattice-Valued Propositional Logic, Gradational Lattice-Valued Propositional Logic, rule of inference, syntax, semantics

\section{Introduction}

In order to provide a logical foundation for uncertain information processing theory, especially for the fuzziness, the incomparability uncertain information in the reasoning, $\mathrm{Xu}$ presented the lattice implication algebra by combining lattice and implication algebra in 1993 [1]. After then, he and his research group have established the lattice-valued propositional logic LP(X) and gradational lattice-valued propositional $\operatorname{logic} \mathrm{L}_{\mathrm{vpl}}$, lattice-valued first-order logic and gradational latticevalued first-order logic and applied them to automated and approximated reasoning [2]-[12]. As we know, during the uncertainty reasoning process based on $\mathrm{L}_{\mathrm{vpl}}$ the chosen of the value set $\mathcal{T}$ and the implication operator $\rightarrow$ is very important. Chen researched uncertainty reasoning based on lattice-valued firstorder logic $\mathrm{L}_{\mathrm{vfl}}$ and give some concert methods for selecting appropriate parameters during the uncertainty reasoning process based on $\mathrm{L}_{\mathrm{vfl}}$ for some representative uncertainty reasoning models[9]. In [9] Chen defined

$$
\begin{aligned}
R^{*} & =\left\{\left(r_{2}^{0}, t_{2}^{*}\right),\left(r_{2}^{*}, t_{2}^{*}\right),\left(r_{2}^{\square}, t_{2}^{*}\right)\right\} \cup\left\{\left(r_{1}^{\theta_{0}}, t_{1}^{\theta_{0}}\right) \mid \theta_{0} \in L\right\} \\
& \cup\left\{\left(r_{1}^{u}, t_{1}\right) \mid u \in U\right\} \cup\left\{\left(r_{2}^{u}, t_{1}\right) \mid u \in U\right\} \\
& \cup\left\{\left(r_{3}^{u}, t_{1}\right) \mid u \in U\right\} \cup\left\{\left(r_{4}^{u}, t_{1}\right) \mid u \in U\right\} \subseteq \Re
\end{aligned}
$$

and $I_{i}=\left\{T \mid T\right.$ is $\alpha$-i type closed w.r.t. $\left.R^{*}\right\}$.

where,

$$
\begin{aligned}
& r_{2}^{0}(\varphi, \varphi \rightarrow \psi)=\psi, t_{2}^{*}(\theta, \beta)=\theta \wedge \beta, \\
& r_{2}^{*}(\varphi \rightarrow \gamma, \varphi \rightarrow \psi)=\varphi \rightarrow(\gamma \wedge \psi), \\
& r_{2}^{\square}(\varphi \rightarrow \psi, \psi \rightarrow \gamma)=\varphi \rightarrow \gamma, \\
& r_{1}^{\theta_{0}}(\varphi)=\theta_{0} \rightarrow \varphi, t_{1}^{\theta_{0}}(\alpha)=\theta_{0} \rightarrow \alpha \\
& r_{1}^{u}(\varphi)=\left(Q_{u} x\right) \varphi, t_{1}(\theta)=\theta, \\
& r_{2}^{u}(\varphi \rightarrow \psi)=\varphi \rightarrow\left(Q_{u} x\right) \psi, x \text { is not free in } \varphi, \\
& r_{3}^{u}(\varphi \rightarrow \psi)=\left(Q_{u} x\right) \varphi \rightarrow \psi, x \text { is not free in } \psi, \\
& r_{4}^{u}\left(Q_{u} x\right)(\varphi \otimes \psi)=\left(Q_{u} x\right) \varphi \otimes \psi, x \text { is not free in } \psi,
\end{aligned}
$$

But we don't know why $\mathscr{R}^{*}$ is chosen as this and what the function of $R^{*}$ is.

\section{Comparing the corresponding definitions}

In the following we suppose the sets $\mathscr{F}$ and $\mathscr{F}_{p}$ are the sets of formula of $L P(X)$ and $L_{v p l}$ respectively. By comparing the definitions of $\operatorname{LP}(\mathrm{X})$ with the counterpart of $\mathrm{L}_{\mathrm{vpl}}$, the meaning of the parameters of $\mathrm{L}_{\mathrm{vpl}}$ is understood.

Definition 2.1 [2] A mapping $v: \operatorname{LP}(\mathrm{X}) \rightarrow \mathrm{L}$ is called a valuation of $\mathrm{LP}(\mathrm{X})$, if it is a T-homomorphism.

Corollary 2.1 [10] Let $f: \mathrm{LP}(\mathrm{X}) \rightarrow \mathrm{L}$ be a mapping of $\operatorname{LP}(\mathrm{X})$, then $f$ is a valuation of $\mathrm{LP}(\mathrm{X})$ if and only if it satisfies
(1). $f(\alpha)=\alpha$ for any $\alpha \in L$;
(2). $f\left(p^{\prime}\right)=(f(p))^{\prime}$ for any $p \in \mathscr{F}$;
(3). $f(p \rightarrow q)=f(p) \rightarrow f(q)$ for any $p, q \in \mathscr{F}$. 
Definition 2.2 [4] Let $\mathscr{F}_{L}(\mathscr{F}), v$ be a valuation of $\mathrm{LP}(\mathrm{X})$. It is called that $v$ satisfies $A$ if $A(p) \leq v(p)$ for any $p \in \mathscr{F} . A$ is called satisfiable if there exists a valuation $v$, which satisfies $A$.

Definition 2.3 [6] Let $X \in \mathscr{F}_{L}\left(\mathscr{F}_{P}\right), \delta \in L$, $T_{0} \in \mathcal{T}$. If for any $P \in \mathscr{F}_{P}$,

(1). $T_{0}\left(p^{\prime}\right)=\left(T_{0}(p)\right)^{\prime}$,

(2). $X(p) \rightarrow T_{0}(p) \geq \delta$

then $T_{0}$ is called $\delta$-i type satisfies $X$. If there exists $T_{0}$, $T_{0} \delta$-i type satisfies $X$, then $\mathrm{X}$ is said to be $\delta$-i type satisfiable, $\mathrm{i}=\mathrm{I}$, II.

Definition 2.4 [4] Let $A \in \mathscr{F}_{L}(\mathscr{F}), A$ is called closed if

(1). $A(p \rightarrow q) \otimes A(p) \leq A(q)$,

(2). $\alpha \rightarrow A(p) \leq A(\alpha \rightarrow p)$.

for any $p, q \in \mathscr{F}$ and $\alpha \in L$.

Definition 2.5 [6] Let $X \in \mathscr{F}_{L}\left(\mathscr{F}_{P}\right),(r, t) \in \mathscr{R}_{n}$, $n \in \square, \alpha \in L$. If

$$
X \circ r \supseteq \alpha \otimes\left(t \circ \prod^{n} X\right)
$$

in $\mathrm{Dn}(\mathrm{r})$, then $X$ is said to be $\alpha$-I type closed w.r.t. $(\mathrm{r}, \mathrm{t})$. If

$$
X \circ r \supseteq t \circ\left(\prod^{n} \alpha \otimes X\right)
$$

in $\operatorname{Dn}(\mathrm{r})$, then $X$ is said to be $\alpha$-II type closed w.r.t. (r, t).

In definition $2.5 \alpha$ denote the consistency of the semantics and syntax When $\alpha=I$, and $\mathscr{R}=\left\{\left(r_{2}^{0}, t_{2}^{0}\right),\left(r_{1}^{\theta}, t_{1}^{\theta}\right)\right\}$, the formula (1), (2) are changed into $\quad X(p \rightarrow q) \otimes X(p) \leq X(q)$, $\alpha \rightarrow X(p) \leq X(\alpha \rightarrow p)$ respectively, which is the same as Definition 2.4. In another word in $\operatorname{LP}(\mathrm{X})$ " $A$ is closed" is to be said for $\left(r_{2}^{0}, t_{2}^{0}\right),\left(r_{1}^{\theta}, t_{1}^{\theta}\right)$ these two inference rules.

\section{Semantics of $\mathrm{LP}(\mathrm{X})$ and $\mathrm{L}_{\mathrm{VPL}}$}

Definition 2.6 [4] Let $A \in \mathscr{F}_{L}(\mathscr{F}), p \in \mathscr{F}$ and $\alpha \in L$. $p$ is called sematically implied from $A$ with truth value level $\alpha$ if $v(p) \geq \alpha$ for any valuation $v$, which satisfies $A$ of $\operatorname{LP}(\mathrm{X})$. We write this $A \mid={ }_{\alpha} p . p$ is called valid with truth value level $\alpha$ (shortly for $\alpha$-valid) and denoted by $\mid={ }_{\alpha} p$, if $v(p) \geq \alpha$ for any valuation $v$ of $\mathrm{LP}(\mathrm{X}) \cdot p$ is called a valid formula, if $\mid=_{I} p \cdot p$ is called $\alpha$-valid in $A$, If $\alpha=\wedge\{v(p) \mid \mathrm{v}$ is a valuation and satisfies $\mathrm{A}\}$ We shall write this $A \|={ }_{\alpha} p$.

$\operatorname{Con}(A)(p) \square \wedge\{v(p) \mid v$ is a valuation of $L P(X)$ and satisfies $A\}$. $\operatorname{Con}(A)(p)$ denote the degree of $p$ can be semantically implied from $A$.

The corresponding semantics of $L_{v p l}$ is given as a mapping $C_{\mathscr{g}}^{X}(p)$.

\section{Definition 2.7 [6]}

$$
\begin{aligned}
C_{\mathscr{J}}^{X}(p) & \square \underset{T \in \mathscr{T}}{\wedge}[\pi(X \subseteq T) \rightarrow T(p)] \\
= & \wedge \underset{T \in \mathscr{T}}{\wedge}\left[\wedge_{q \in \mathscr{F}_{P}}(X(q) \rightarrow T(q)) \rightarrow T(p)\right] .
\end{aligned}
$$

We can see this operator is a semantic operator of $\mathrm{L}_{\mathrm{vpl}} \cdot C_{\mathcal{g}}^{X}(p)$ denotes the degree of $p$ can be semantically implied from $X$.

Theorem 2.1 [10] Each valuation of $L P(X)$ is closed.

Theorem 2.2 Let $A$ be closed. Then $A(\alpha) \leq \alpha$, and $A\left(p^{\prime}\right) \leq A(p)^{\prime}$ for any $\alpha \in L$ and $p \in \mathscr{F}$.

Proof. $A$ is closed so for any $\alpha \in L$ and $p \in \mathscr{F}$ we have

$$
\alpha \rightarrow A(p) \leq A(\alpha \rightarrow p) \leq A(\alpha) \rightarrow A(p)
$$

Hence $A(\alpha) \leq \alpha$, and $A(0)=0$.

$$
\begin{aligned}
A\left(p^{\prime}\right) & =A(p \rightarrow 0) \leq A(p) \rightarrow A(0) \\
= & A(p) \rightarrow 0=A(p)^{\prime} .
\end{aligned}
$$

Theorem 2.3 If $\varnothing \neq \mathfrak{A} \subseteq\{\mathrm{A} \mid \mathrm{A}$ is closed $\}$, then $\cap_{A \in \mathfrak{A} A} A$ is closed.

Proof. For any $\alpha \in L$ and $p \in \mathscr{F}$

$$
\begin{aligned}
& \cap_{A \in \mathfrak{A}} A(q)=\wedge_{A \in \mathbb{A}} A(q) \geq \wedge_{A \in \mathbb{A}}(A(p) \otimes A(p \rightarrow q)) \\
& \geq \wedge_{A \in \mathbb{A}} A(p) \otimes \underset{A \in \mathbb{A}}{\wedge} A(p \rightarrow q) \\
& =\cap_{A \in \mathfrak{A}} A(p) \otimes \cap_{A \in \mathfrak{A}} A(p \rightarrow q) . \\
& \left(\cap_{A \in \mathfrak{A}} A\right)(\alpha \rightarrow p) \geq \underset{A \in \mathfrak{A}}{\wedge}(\alpha \rightarrow A(p)) \\
& \geq \alpha \rightarrow \underset{A \in \mathbb{A}}{\wedge} A(p) \\
& =\alpha \rightarrow\left(\cap_{A \in \mathfrak{A}} A\right)(p) .
\end{aligned}
$$

Corollary 2.2 If $A\left|=_{\alpha} p, A\right|=_{\beta} p \rightarrow q$, then

(1). $A \mid={ }_{\alpha \otimes \beta} q$,

(2). $A \mid={ }_{\alpha_{0} \rightarrow \alpha} \alpha_{0} \rightarrow p$ for any $\alpha_{0} \in L$.

\section{Proof.}

$\operatorname{Con}(A)(p) \square \wedge\{v(p) \mid v$ is a valuation of $L P(X)$

and satisfies $A\}$, so $\operatorname{Con}(A)$ is closed.

Hence $\operatorname{Con}(A)(p \rightarrow q) \otimes \operatorname{Con}(A)(p) \leq \operatorname{Con}(A)(q)$, $\alpha_{0} \rightarrow \operatorname{Con}(A)(p) \leq \operatorname{Con}(A)\left(\alpha_{0} \rightarrow p\right) \quad$ for any 
$p, q \in \mathscr{F}$ and $\alpha_{0} \in L$. If $A\left|=_{\alpha} p, A\right|=_{\beta} p \rightarrow q$, i.e. $\alpha \otimes \beta \leq \operatorname{Con}(A)(p \rightarrow q) \otimes \operatorname{Con}(A)(p) \leq \operatorname{Con}(A)(q)$, $\alpha_{0} \rightarrow \alpha \leq \alpha_{0} \rightarrow \operatorname{Con}(A)(p) \leq \operatorname{Con}(A)\left(\alpha_{0} \rightarrow p\right)$ hence $A\left|=_{\alpha \otimes \beta} q, A\right|=_{\alpha_{0} \rightarrow \alpha} \alpha_{0} \rightarrow p$ for any $\alpha_{0} \in L$.

\section{Syntax of $L P(X)$ and $L_{V P L}$}

Definition 2.8 [5] Let $A \in \mathscr{F}_{L}(\mathscr{F}), p \in \mathscr{F}$. A formal proof $\omega$ of $p$ from $A$ is a finite sequence as follows:

$\left(p_{1}, \alpha_{1}\right), \ldots,\left(p_{n}, \alpha_{n}\right)$,

where $p_{n}=p$, and for any $i, 1 \leq i \leq n, p_{i} \in \mathscr{F}, a_{i} \in L$ and

(1). $A_{L}\left(p_{i}\right)=\alpha_{i}$ or

(2). $A\left(p_{i}\right)=\alpha_{i}$, or

(3). there exist $j, k<i$, such that $p_{j}=p_{k} \rightarrow p_{i}$ and $a_{i}=a_{j} \otimes a_{k}$ or

(4). there exists $j<i$ and $\alpha \in L$, such that $p_{i}=\alpha \rightarrow p_{j}$ and $\alpha_{i}=\alpha \rightarrow \alpha_{j}$.

Where the operation $\otimes$ is defined as $\left(\alpha \rightarrow \beta^{\prime}\right)^{\prime}$ in $L$.

Definition 2.9 [5] Let $A \in \mathscr{F}_{L}(\mathscr{F}), p \in \mathscr{F}$, $\alpha \in L \cdot p$ is called an $\alpha$-theorem of $A$ and written as $A$ $\mid-{ }_{\alpha} p$, if $\alpha \leq \vee\{\operatorname{val}(w) \mid w$ is a proof of $\mathrm{p}$ from $A\}$.

If $\alpha=\vee\{\operatorname{val}(w) \mid w$ is a proof of $\mathrm{p}$ from $A\}$,

then it is written as $A \|{ }_{\alpha}$ p.

In [10] define a mapping

Ded: $\mathscr{F}_{L}(\mathscr{F}) \rightarrow \mathscr{F}_{L}(\mathscr{F})$ satisfy

$\operatorname{Ded}(A)(p)=\vee\{\operatorname{val}(w) \mid w$ is a proof of $\mathrm{p}$ from $A\}$

for any $p \in \mathscr{F}$ and $A \in \mathscr{F}_{L}(\mathscr{F})$.

$\operatorname{Ded}(A)(p)=\vee\left\{B(p) \mid \mathrm{B} \supseteq \mathrm{A} \cup \mathrm{A}_{L}, B\right.$ is closed $\}$.

So $\operatorname{Ded}(A)(p)$ denote the degree of $p$ syntactically from $A$.

Definition

\subsection{0}

[6]

Let $X \in \mathscr{F}_{L}\left(\mathscr{F}_{P}\right), \mathscr{T} \subseteq \mathscr{F}_{L}\left(\mathscr{F}_{P}\right), p \in \mathscr{F}_{P}, \theta, \alpha, \beta \in L$. $\left(P^{I},(n), X,(p, \theta)-(\alpha, \beta)\right)$ is said to be a $(\alpha, \beta)-I$ type proof with the truth degree $\theta$ from $X$ to $p$ (shortly, $\theta-(\alpha, \beta)$-I type proof from $X$ to $p$ ), if the mapping

$\mathrm{P}^{\mathrm{I}}:(\mathrm{n}) \rightarrow \mathscr{F}_{P} \times \mathrm{L}((\mathrm{n})=\{1,2, \cdots, \mathrm{n}\})$,

$\mathrm{i} \rightarrow\left(\mathrm{p}_{\mathrm{i}}, \theta_{\mathrm{i}}\right)$,

satisfies

(1). $\left(\mathrm{p}_{\mathrm{n}}, \theta_{\mathrm{n}}\right)=(\mathrm{p}, \theta)$ and

(2). $\theta_{\mathrm{i}}=\beta \otimes C_{\mathcal{J}}^{\varnothing}\left(p_{i}\right)$, or

(3). $\theta_{\mathrm{i}}=\beta \otimes X\left(p_{i}\right)$, or
(4). there exist $\mathrm{i}_{1}, \cdots, \mathrm{i}_{\mathrm{k}} \leq \mathrm{i}$, and $(\mathrm{r}, \mathrm{t}) \in \mathscr{R}_{k}$ such that $\left(\mathrm{p}_{\mathrm{i}}, \theta_{\mathrm{i}}\right)=\left(\mathrm{r}\left(\mathrm{p}_{\mathrm{i} 1}, \cdots, \mathrm{p}_{\mathrm{ik}}\right), \alpha \otimes \mathrm{t}\left(\theta_{\mathrm{i} 1}, \cdots, \theta_{\mathrm{ik}}\right)\right)$,

where $\mathrm{n}$ is said to be the length of $\theta-(\alpha, \beta)$-I type proof from $X$ to $p$ under $\mathrm{P}^{\mathrm{I}}$, and denoted as $l\left(\mathrm{P}^{\mathrm{I}}\right)$.

$\theta-(\alpha, \beta)$-II type proof from $X$ to $p$ is the same as definition 2.10 except for (4) changed into

$\left(\mathrm{p}_{\mathrm{i}}, \theta_{\mathrm{i}}\right)=\left(\mathrm{r}\left(\mathrm{p}_{\mathrm{i} 1}, \cdots, \mathrm{p}_{\mathrm{ik}}\right), \mathrm{t}\left(\alpha \otimes \theta_{\mathrm{i} 1}, \cdots, \alpha \otimes \theta_{\mathrm{ik}}\right)\right)$.

Definition 2.11 [6]

$C_{\left(C_{\mathcal{T}}^{\beta,}, \mathcal{R}(\alpha-i)\right)}^{\beta, X} \square \wedge\left\{Y(p) \mid Y \supseteq \beta \otimes\left(C_{\mathcal{I}}^{\varnothing} \cup X\right)\right.$,

$Y$ is $\alpha$-i type closed w.r.t.R\} .

Theorem 2.4 [6] Let $X \in \mathscr{F}_{L}\left(\mathscr{F}_{P}\right), \mathscr{T} \subseteq \mathscr{F}_{L}\left(\mathscr{F}_{P}\right)$, $\alpha, \beta \in L$, and the truth-valued operations in $\Re$ satisfy finite semicontinuity, then for any $p \in \mathscr{F}_{P}$,

$C_{\left(C_{\mathcal{J}}^{\beta, R(\alpha-i))}\right.}^{\beta, X}=\vee\left\{\theta\right.$ there exists $\left.\left(P^{i},(n), X,(p, \theta)-(\alpha, \beta)\right)\right\}$ for $\mathrm{i}=\mathrm{I}$, II.

From Theorem 2.4 we know when the truth-valued operations in $R$ satisfy finite semicontinuity $C_{\left(C_{\mathcal{F}, R(\alpha-i))}^{\beta, X}\right.}^{\beta, X}$ is a syntactic operator of $\mathrm{L}_{\mathrm{VPL}}$, and $C_{\left(C_{\mathcal{T}}^{\otimes}, \mathbb{R}(\alpha-i)\right)}^{\beta, X}(p)$ denote the degree of $P$ can be syntactically implied from $X$.

\section{The Relations between $\operatorname{LP}(X)$ and $L_{V P L}$ and the role of inference rule set $R$ playing in uncertainty reasoning based on $L_{v p l}$}

We know any logic system is composed of two parts the semantics and the syntax. So we can discuss the relations of these two logic systems by discussing the relations between the semantics and the syntax of $\mathrm{LP}(\mathrm{X})$ and $\mathrm{L}_{\mathrm{VPL}}$. In $\mathrm{L}_{\mathrm{VPL}} \alpha$ denote the consistency of semantics and syntax, and $\beta$ denote the degree of truth value deliver [11]. Firstly, we discuss the semantics of these two logic systems.

$$
\begin{aligned}
C_{\mathcal{I}}^{X}(p) & \underset{T \in \mathcal{T}}{\wedge}[\pi(X \subseteq T) \rightarrow T(p)] \\
& =\underset{T \in \mathcal{T}}{\wedge}\left[\underset{q \in \mathscr{F}_{P}}{\wedge}(X(q) \rightarrow T(q)) \rightarrow T(p)\right],
\end{aligned}
$$

when $\alpha=\beta=I, \Re^{0}=\left\{\left(r_{2}^{0}, t_{2}^{0}\right),\left(r_{1}^{\theta}, t_{1}^{\theta}\right)\right\}$, and $\mathscr{T}^{\circ}=\left\{T \mid T: \mathscr{F}_{P} \rightarrow L\right.$ is a homomorphic mapping and $I-i$ satisfies $X\}, i=\mathrm{I}$, II .

For any $T \in \mathcal{T}^{\circ} \pi(X \subseteq T)=I$ i.e. for any $T \in \mathcal{T} T$ I-i type satisfies $X$. Here $C_{\mathcal{T}}^{X}(p)=\underset{T \in \mathcal{T}}{\wedge}\{T(p)\}$ In $\mathrm{LP}(\mathrm{X})$ the semantic operator 
$\operatorname{Con}(A)(p)$

$\square \wedge\{v(p) \mid v$ is a valuation of $\mathrm{LP}(\mathrm{X})$ and satisfies $\mathrm{A}\}$.

Hence these two semantic operator coincide.

In the following we discuss the syntax of these two logic systems.

By comparing Definition 2.8 with Definition 2.10 we can see that the difference of these two definitions contains these three aspects.

(1) In $\mathrm{L}_{\mathrm{VPL}}$ the consistency of semantics and syntax is $\alpha$, while in $\mathrm{LP}(\mathrm{X})$ the consistency of semantics is $I$;

(2) The degree of truth value deliver is $\beta$ while in $\mathrm{LP}(\mathrm{X})$ the degree is $I$;

(3) The inference rule set of LP(X) only contain $\left(r_{2}^{0}, t_{2}^{0}\right),\left(r_{1}^{\theta}, t_{1}^{\theta}\right)$, while in $\mathrm{L}_{\mathrm{VPL}}$ the inference rule set contains more.

From the above discussion we can say that $\mathrm{L}_{\mathrm{VPL}}$ is the generalization of $\mathrm{LP}(\mathrm{X})$, with its semantic and syntax is $\alpha$ consistent, truth-value transition degree is $\beta$, and inference rule is much more which determines the syntactic function of $\mathrm{L}_{\mathrm{VPL}}$ is stronger.

The gradational lattice-valued propositional logic $\mathrm{L}_{\mathrm{VPL}}$ denotes a series of logic systems. Different parameters determine different logic system. We discuss the function of inference rule set $R$ in $L_{V P L}$ as follows.

$\begin{array}{llll}\text { Theorem } & 3.1 & \text { Let } \quad R_{1} \subseteq R_{2}\end{array}$, $\mathscr{T}_{i}^{1}=\left\{T \mid T\right.$ is $\alpha-i$ type closed w.r.t. $\left.\mathscr{R}_{1}\right\}$ $\mathscr{T}_{i}^{2}=\left\{T \mid T\right.$ is $\alpha$-i type closed w.r.t. $\left.\Re_{2}\right\} \quad$. i $=\mathrm{I}, \quad$ II. Then

(1). $C_{\left(C_{f_{i}^{2}}^{\beta,}, \mathcal{R}_{2}(\alpha-i)\right)}^{\beta, X} \supseteq C_{\left(C_{f_{i}}^{\beta}, \mathcal{R}_{1}(\alpha-i)\right)}^{\beta, X}$,

(2). $C_{\mathscr{f}_{i}^{2}}^{\beta \otimes X} \supseteq C_{\mathscr{f}_{i}^{1}}^{\beta \otimes X}$.

Proof. If $\mathrm{Y}$ is $\alpha-\mathrm{i}$ type closed w.r.t. $\mathfrak{R}_{2}$ and $\Re_{1} \subseteq \Re_{2}$, hence If $\mathrm{Y}$ is $\alpha$-i type closed w.r.t. $\Re_{1}$

$\left\{Y \mid Y\right.$ is $\alpha$-i type closed w.r.t. $\left.\Re_{2}\right\}$ $\subseteq\left\{Y \mid Y\right.$ is $\alpha$-i type closed w.r.t. $\left.\Re_{1}\right\}$

i.e. $\mathscr{J}_{i}^{2} \subseteq \mathfrak{g}_{i}^{1}$, hence $C_{\mathscr{f}_{i}^{2}}^{\varnothing} \supseteq C_{\mathscr{f}_{i}^{1}}^{\varnothing}$

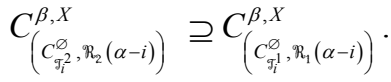

$$
\begin{aligned}
& C_{\mathcal{T}_{i}^{2}}^{\beta \otimes X}=\underset{T \in \mathcal{T}_{i}^{2}}{\wedge}[\pi(\beta \otimes X \subseteq T) \rightarrow T(p)] \\
& \supseteq \underset{T \in \mathcal{T}_{i}^{i^{\prime}}}{\wedge}[\pi(\beta \otimes X \subseteq T) \rightarrow T(p)] . \\
& =C_{\tilde{g}_{i}^{1}}^{\beta \otimes X}
\end{aligned}
$$

In fact, to some extent, the inference rule set $R$ determine the syntactic function of $\mathrm{L}_{\mathrm{vpl}} \cdot \Re$ relates to the valuation set $\mathcal{T}$ closely, because $\mathcal{T}$ must be $\alpha$-i type closed w.r.t. $R$. Furthermore $\mathcal{T}$ and the implication operator $\rightarrow$ determine the semantic function of $\mathrm{L}_{\mathrm{vpl}}$. In the above theorem we can see the more of its number is the stronger of the syntactic function and the semantic function of $\mathrm{L}_{\mathrm{vpl}}$ is. Hence the chosen of $\Re$ is very important.

\section{Conclusions}

By comparing the definitions of $\mathrm{L}_{\mathrm{vpl}}$ with the corresponding definitions of $\mathrm{LP}(\mathrm{X})$ we understand the meaning of the parameters of $\mathrm{L}_{\mathrm{vpl}}$. We conclude that $\mathrm{L}_{\mathrm{vpl}}$ is a generalization of $\mathrm{LP}(\mathrm{X})$. We point out the main three generalized aspects. And we obtain that in $\mathrm{L}_{\mathrm{vpl}}$ the chosen of the inference rule set $R$ is very important. The more its number, the stronger the syntactic function and the semantic function of $\mathrm{L}_{\mathrm{vpl}}$ is. As we know in the uncertainty reasoning based on $\mathrm{L}_{\mathrm{vpl}}$, we have to choose proper valuation set $\mathcal{T}$ s.t. "r: If $X$, then $Y^{\prime \prime}$ is $(\alpha, \beta, \tau, \mathcal{T})$-i type representable. Our further research work are to choose proper inference rule set $R$ and valuation set $\mathcal{T}$.

\section{Acknowledgement}

The work was partially supported by the National Natural Science Foundation of P. R. China (Grant No. 60474022) and the Research Fund for the Doctoral Program of Higher Education (Grant No. 20060613007).

\section{References}

[1] Y Xu. Lattice implication algebras. J. Southwest Jiaotong University, 28 (1): 20-27, 1993 (in Chinese).

[2] Y Xu, K.Y. Qin, Lattice-valued propositional logic (I). J. Southwest Jiaotong University (English Edition), 1(2):123-128, 1993.

[3] K.Y. Qin, Y. Xu, Lattice-valued propositional logic (II), J. Southwest Jiaotong University (English Edition), 2(1):22-27, 1994.

[4] K.Y. Qin, Y. Xu, Lattice-valued propositional logic L(X) (I)*. J. Fuzzy systems and mathematics, 11(4):5-11, 1997 (in Chinese).

[5] K.Y. Qin, Y. Xu, Z.M. Song, Lattice-valued propositional logic L(X) (II)*. J. Fuzzy systems and mathematics, 12(1): 10-19, 1998 (in Chinese).

[6] Y. Xu, K.Y. Qin, J. Liu, et al, L-valued propositional logic $\mathrm{L}_{\mathrm{vpl}}$, J. Inform. Sci., 114: 205235, 1999.

[7] Y. Xu, J. Liu, Z.M. Song, et al, On semantics of L-valued first- order logic $\mathrm{L}_{\mathrm{vfl}}(\mathrm{I})$, Int. J. Gen. Syst., 29: 53-79, 2000. 
[8] Y. Xu, Z.M. Song, K.Y. Qin, et al, Syntax of Lvalued first order logic $\mathrm{L}_{\mathrm{vfl}}$, Int. J. MutipleValued Logic, 7: 213-257, 2001.

[9] Y. Xu, D. Ruan, J. Liu, Approximate reasoning based on lattice-valued propositional logic $\mathrm{L}_{\mathrm{vpl}}$. In: Ruan Da and E.E. Kerre(Eds) Fuzzy If-Then Rules in Computational Intelligence: Theory and Applications. Kluwer Academic Publishers, pp. 81-105, 2000.

[10] Y. Xu, D. Ruan, K.Y. Qin, J. Liu, Lattice-Valued Logic: An Alternative Approach to teat Fuzziness and Incomparability, Germany: Springer-Verlag, 2003.

[11] S.W. Chen, Study on Uncertainty Reasoning in Lattice-Valued First-order Logic Based on Lattice Implication Algebra. Ph.D. Thesis, Southwest Jiaotong Univ., China, 2005.

[12] S.W. Chen, Rules of inference in latticed-valued propositional logic $\mathrm{L}_{\mathrm{vpl}}$, J. Southwest Jiaotong University, 41(2):256-258, 2006 (in Chinese). 